Abmad Mansour und Gürcan Kökgiran

\title{
„Freiheit beginnt im Kopf“ - Präventionsansätze mit ReThink, Reflect und ReStart gegen islamische Radikalisierung. Ein Praxisbericht.
}

\section{Zusammenfassung}

Mündigkeit ist für eine aufgeklärte, plurale Lebensweise und demokratische Gesellschaftsordnung eine sine qua non, also eine unerlässliche Notwendigkeit. Bildung, Erziehung, Familienstrukturen uvm sind darauf ausgerichtet, dass junge Menschen befähigt werden eigenverantwortlich zu handeln und kritisch zu denken. Kollektivistische Gesellschaften, die häufig patriarchale und religiöse Strukturen aufweisen, beruhen auf dem Gehorsam gegenüber Älteren und/oder männlichen Familienmitgliedern, wodurch der Fortbestand des Kollektivs gesichert wird. Kommen Faktoren wie Lebenskrisen, biographische Brüche, Mobbing, das Gefühl der Entfremdung und abgehängt worden zu sein, eine fehlende oder eine gar repressive Vaterfigur hinzu, sind günstige Bedingungen für die Empfänglichkeit radikaler Missionierungsversuche geschaffen. Wie können Jugendliche und junge Menschen vor Radikalisierungsbestrebungen geschützt werden? Welche Instrumentarien der aktuellen Präventionsarbeit stehen hierfür zur Verfügung? Diese Fragen werden in diesem Artikel anhand der WorkshopProjekte „ReStart“, „Reflect“ und „ReThink“ der Mansour-Initiative für Demokratieförderung und Extremismusprävention erörtert.

Keywords: Mündigkeit, Prävention, Deradikalisierung, Demokratieförderung, Islamismus, MIND prevention

„Freedom beginns in your mind" - An approach to prevent islamic radicalisation with ReThink, Reflect and ReStart

\section{Abstract}

Taking personal responsibility is a sine qua non for an enlightened, plural way of life and a democratic social order, that is, an essential necessity. Education, upbringing, family structures and much more are aimed to enable young people to act independently and to think critically. Collectivist societies, which often have patriarchal and religious structures, are based on obedience to the elderly and / or male family members, thereby ensuring the continued existence of the collective. If factors such as life crises, bio- 
graphical breaks, bullying, the feeling of alienation and being left behind, a missing or even a repressive father figure are added, favorable conditions are created for the susceptibility of radical attempts at missionary activity. What preventive measures could help to prepare young people in terms of radicalization efforts and therefore protect them in the long run? The following article refers to these issues including the workshop projekts "ReStart”, „Reflect" and "ReThink” of the Mansour Initiative for Democracy Promotion and Extremism Prevention in this discussion.

Keywords: personal responsibility, prevention, de-radicalization, democracy promotion, islamism, MIND Prevention

\section{Einleitung}

Irgendwo in Deutschland. Ahmad Mansour sitzt gemeinsam mit seinem Team und 20 nervösen Männern im Kreis in einem kleinen Raum. Die kleinen Fenster sind vergittert, durch sie fällt diffuses Licht. Draußen im verschneiten Hof laufen Männer in Parkas im Kreis, rauchen und unterhalten sich. Die Gruppe befindet sich im Kellergeschoss eines Gefängnisses. Mansour arbeitet mit seiner 2017 gegründeten Initiative ${ }^{1}$ mit verschiedenen Bundesländern zusammen, entwickelt und setzt Konzepte um. Dieses Projekt, durchgeführt in bayerischen Haftanstalten, heißt ReStart ${ }^{2}$, er ist heute mit drei Kollegen hier, um mit den Inhaftierten einen drei-stündigen Workshop durchzuführen. An der Tafel stehen noch Matheaufgaben. Aber heute geht es nicht um Gleichungen, heute geht es um Gleichnisse. Es werden keine leichten drei Stunden werden - für keinen der Beteiligten.

Die Gruppe besteht aus Menschen mit häufig schwierigen Lebensverläufen, die in ihrer persönlichen Krise, der Inhaftierung, nach Halt und Orientierung suchen. Sie sind besonders anfällig für radikale Gedanken, die ihnen Heil durch Allahs Rehabilitation versprechen. Mansours Initiative aber möchte mit ihren Projekten schneller sein als die Radikalen aller Couleur. Sein Team und er möchten reden und aufklären, jedem die Möglichkeit geben für einen selbstbestimmten Neubeginn - im Sinne von Aufklärung und Demokratie. Keiner der Angesprochenen soll aus Hoffnungslosigkeit und wegen starrer Ansichten in die Radikalisierung laufen.

Das geflügelte Motto für die Projekte der MIND prevention lautet „Freiheit beginnt im Kopf“ und ist programmatisch. Während sich die Projekte ReThink und Reflect auf

1 MIND prevention = Mansour-Initiative für Demokratieförderung und Extremismusprävention

2 Kurze Beschreibung, wofür ReStart steht. ReStart richtet sich an Insassen der bayerischen Justizvollzugsanstalten mit dem Ziel religiös begründeter Radikalisierung präventiv zu begegnen. Durch Workshops und Rollenspiele soll kritisches Denken geschult werden, um Einstellungen und Meinungen zu den Themen wie Islamverständnisse, Gleichberechtigung und Männlichkeitskonzept zu hinterfragen und neu zu bewerten. In einer zweiten Ebene des Projektes richten sich Fortbildungsangebote an Beschäftigte im Bereich des Justizvollzugs, die zu professionellem Handeln in Bezug auf islamistische Radikalisierung unter Strafgefangenen befähigen sollen. Das Projekt wird in Kooperation mit dem Bayerischen Staatsministerium der Justiz und für Verbraucherschutz durchgeführt. 
Schulen und Geflüchtete Gruppen konzentrieren, setzt ReStart in den Jugendvollzugsanstalten an. Gerade Gefängnisse sind ein wichtiger Ort für Präventionsarbeit. In der Haft befinden sich Jugendliche in der Regel in einer persönlichen Krise, sie haben die Anerkennung der Gesellschaft verloren, werden in ihrem Umfeld und in der Familie stigmatisiert, manchmal sogar ausgestoßen. Sie sind allein mit ihren Sorgen, haben viel Zeit und suchen meistens nach einem neuen Anfang. Durch die Ereignisse der letzten Jahre, wie Verhaftungen von Rückkehrern und Radikalen, hat die Zahl inhaftierter Islamisten zugenommen und somit ist in den Haftanstalten auch die Zahl der Missionierungsversuche gestiegen. Außerdem zeigen die Radikalisierungsverläufe der Attentäter in Berlin, Paris und Straßburg, dass während ihrer Haftzeiten zur kriminellen eine ideologische Dimension kam, die in ihrer Mischung fatale Folgen nach sich zogen. ${ }^{3}$

Die Projekte „ReStart“, „ReThink“" und „Reflect“ schaffen in Workshops interaktive Räume, in denen Dialoge stattfinden, ohne Verurteilung, ohne Belehrung, ohne Unterrichtsatmosphäre. Dieser Rahmen ermöglicht den Jugendlichen und jungen Menschen frei zu sprechen, ihre Meinung zu äußern und eine emotionale Reise mit den Workshop-Leitern anzutreten, zu Themen, die sie entmündigen und gleichzeitig so anfällig für die radikale Ideologie machen. Das Setting der Workshops ist so angelegt, dass Rollenspiele Denkanstöße gegeben, um Wertevorstellungen und Meinungen zu Themen wie Männlichkeit und Gleichberechtigung, Islamverständnis, patriarchale Strukturen, Verschwörungstheorien und Antisemitismus kritisch zu hinterfragen.

Das Ziel ist, genau die Themen zu besprechen, die Radikale ansonsten für sich beanspruchen, über ihre Weltbilder und Einstellungen kritisch zu reflektieren und im günstigsten Fall ihre Haltungen zu überdenken. Die Adressaten sind zwischen 16 und 60 Jahre alt, eine heterogene Truppe aus Herkunftsländern wie Libanon, Afghanistan, Serbien, Marokko, Irak, Türkei, Tschechien, Deutschland oder Irland. Sie haben unterschiedliche Konfessionen, sind Christen, Moslems, Juden oder Atheisten.

Das Team von MIND prevention versteht sich nicht als die Polizei, nicht als die Justiz oder als eine moralische Instanz, sie sind nicht die „anderen“, vielmehr sprechen sie oft die gleiche Sprache, kennen die gleichen soziokulturellen Codes, stammen aus ähnlichen Milieus, haben selbst Migrationshintergrund und manchmal sogar die gleichen Namen, das alleine schafft schon in der Vorstellungsrunde ein Grundvertrauen. Sie müssen niemandem etwas vormachen, schließen niemanden aus, sind im Hinblick auf ihre Taten und Meinungen nicht normativ wertend. Vielmehr ist das methodische Set-

3 https://www.dw.com/de/kampf-gegen-islamismus-im-gef\%C3 \% A4ngnis/a-49860809, aufgerufen am 6.2.2020.

4 Das Projekt ReThink will in seinen Workshops für junge, geflüchtete Menschen einen Raum des Dialogs auf Augenhöhe schaffen, indem es den Teilnehmenden gelingt, ihre Werte, Einstellungen und Meinungen zu den Themen Gleichberechtigung, Männlichkeitskonzept, Islamverständnis und Antisemitismus zunächst frei zu formulieren. Durch Anregungen, Interventionen und Reflexion durch die Workshop-Leiter soll erreicht werden, dass die Teilnehmenden lernen, ihre bisherigen Einstellungen zu diskutieren, in Frage zu stellen, Ängste abzubauen und neue Denkanstöße auch in ihre peer-groups und Familien mitzunehmen. Die Workshop-Leiter sollen mit ihrer eigenen Biographie und Persönlichkeit Vorbilder aus der eigenen Community darstellen, wie man als Migrant und Muslim in Deutschland ankommen kann.

RPsych 6. Jg. 1/2020 
ting des Projektes darauf ausgelegt, drei Stunden lang einen gemeinsamen Raum zu schaffen, in dem die Beteiligten mit ihren Gedanken ungezwungen frei sein können, gemäß dem Motto: „Freiheit beginnt im Kopf“.

Ablauf und Anordnung der Workshops sind nicht allein auf die starre Durchführung der pädagogischen Zielsetzung fokussiert, es steht hier nicht das Team und dort diejenigen, die es zu therapieren gilt. Dieses Gefühl soll vermieden werden. Das Team demonstriert unterschiedliche Meinungen, streitet sich auch und macht zwischen all den ernsten Themen gelegentlich Späße, das lockert die Atmosphäre ungemein, regt die Gedanken an, viele der Teilnehmer trauen sich dann, frei zu reden.

Das Team rund um Ahmad Mansour ist also lediglich Begleiter einer Reise, die die Teilnehmer selbst steuern. Sie verstehen sich als Gedankenpflanzer im Garten der Mündigkeit und Aufklärung. Dabei wollen sie ihnen weder etwas eintrichtern noch etwas vorschreiben. Das einzige Ziel ist, sie zum Nachdenken anzuregen über ihre grundsätzlichen Ansichten, die sie mutmaßlich nie hinterfragt haben oder über die sie noch niemals nachgedacht haben. Hier geht es nicht um die Durchsetzung von Direktiven, um richtig und falsch, das ist nicht der methodische Ansatz. Der Dialog steht im Vordergrund und er soll auf Augenhöhe stattfinden und nicht erwachsene Menschen mit erhobenem Zeigefinger resozialisieren, gar disziplinieren, ihnen zeigen, wo es langgeht, denn genauso kennen es viele dieser Männer. Sie sind damit groß geworden.

Rollenspiele, die die Themen für Diskussionen öffnen, sind hierfür ein wirkmächtiges Instrument in den Projekten ReThink, Reflect und ReStart. Dabei sind diese Rollenspiele keinesfalls geschlossene Geschichten mit Moral, sondern sie spiegeln Situationen aus dem Alltag dieser Menschen wider. Während der Diskussion mit den Teilnehmern ermutigen die Workshop-Leiter diese, alternative Verläufe zu entwickeln. Das gibt Mut, selber zu denken und zu handeln.

Die Pädagogen und Psychologen im Team sind vorbereitet darauf, dass manche der Angesprochenen die Augenhöhe überfordern wird, sie wissen, wie schwer es in bestimmten soziokulturellen Kontexten ist, eigene Standpunkte und Entscheidungen zu vertreten. Sie sind auf schwierige emotionale Momente gefasst, in denen die gemeinsame Reflektion zu schmerzhaften Erkenntnissen darüberführt, warum sie im Gefängnis gelandet sind. Aber auch darauf, dass sie bei manchen leider überhaupt nichts erreichen werden. Der Workshop beginnt und Mansour und seine Kollegen stellen sich vor:

"Ich bin Abmad. Können wir uns duzen?" Die Männer nicken. Dann stellen sich die Jungen und Männer der Reihe nach vor. Ein jüngerer mit kurzen, gegelten Haaren, der die ganze Zeit unrubig mit den Beinen wippt und schnell spricht, sagt zum Beispiel: "Ich heiße Arwin, bin aus Afghanistan. Ich bin 16. ". Ein anderer, älterer, grane Haare, Zabnlücke, unrasiert mit Brille, sagt: "Ich heiße auch Abmad. Lustig. Ich komme aus Libyen, bin 45. Ach, ich bin übrigens unschuldig hier. Ich antworte: "Es interessiert uns nicht, warum ibr im Gefängnis seid. Und wir versprechen euch nicht, dass ihr morgen aus dem Gefängnis gehen könnt, weil ibr hier mitmacht. Wir versprechen euch nur eines: ench neue Wege aufzuzeigen. Aber der Weg ist eurer. Den müsst ibr alleine gehen. Wir werden ein paar Rollenspiele mit euch machen und euch 
dabei keine Regeln vorgeben, außer der, dass wir alle respektvoll miteinander umgeben. Schaffen wir das? "Die Männer nicken wieder. Das mit dem Respekt zu erwähnen ist notwendig, denn Respekt ist im Gefängnis keine Selbstverständlichkeit. Trotzdem klappt es immer, dass der Ton zwar manchmal laut, aber nicht verletzend oder grenzüberschreitend ist.

Anschließend fangen die Rollenspiele an. Die erste Szene beschreibt überspitzt eine familiäre Situation. Ein Vater kommt nach Hause und sieht seinen Sobn vor der Spielekonsole, er zockt. Wutentbrannt gibt er seinem Sobn einen Schlag auf den Hinterkopf:

"Was machst du bier? Spielen, spielen, spielen! Den ganzen Tag spielst du. Gebst nicht in die Schule, sitzt nur zu Hause rum und machst nichts, außer zu spielen."

"Ja, aber Baba..."

"Deine Mutter weint jeden Tag wegen dir. Und warst du beute nicht in der Moschee? Alle fragen mich ständig nach dir. Und ich? Ich weiß nie, was ich sagen soll. Ich schäme mich."

»Ich weiß auch nicht ..."

"Ich weiß auch nicht, ich weiß auch nicht! Was weißt du denn, Sobn? Sag's mir!"

$» I c h \ldots \ll$

»Was?«

»Mir gebt's nicht so gut ..."

"Kein Wunder. Du hängst ja auch nur zu Hause rum und spielst. Zur Schule geben sollst $d u$, arbeiten, beten sollst $d u$, auf deine Schwestern aufpassen. Nichts davon machst du. Was soll nur aus dir werden? Eine Schande bist du!"

»Baba,ich..."

"Sei rubig. Ich will nichts mehr hören. Geb mir aus den Augen. Sofort."

Alle applaudieren nach dem Rollenspiel. Auf die Frage „Was fällt euch dazu ein?" kommen manche der sebr unterschiedlichen Antworten unmittelbar, andere sebr zögerlich: "Ha, genauso ist mein Vater. Als ob ibr in meiner Familie zu Besuch wart." Ein anderer: „Ich wünschte, ich hätte so einen Vater gehabt. Meiner hat mich eigentlich immer nur ignoriert. Da konnte ich machen, was ich wollte. "Und wiederum ein anderer beschreibt sein Verhältnis zum Vater: „Ich verstehe den Vater. Er will dem Sobn etwas vermitteln. Da muss man auch streng sein. Die Eltern meinen es ja nicht böse. Sie wollen, dass man etwas von der Familie, Religion und Tradition lernt."

Zunächst erhält die Vaterrolle von den Gefangenen häufig viel Zuspruch. Mansour richtet sich an seinen Kollegen, der die Vaterrolle spielt: „Was bist du für ein Vater? Wo ist die Liebe? Hast du dich gefragt, wie es dazu kommen konnte, dass dein Sobn jetzt so ist?" Sein Kollege in der Vaterrolle antwortet: „Ich gebe jeden Tag zwölf 
Stunden arbeiten, nur für ibn, das ist doch Liebe!" Dann fragt Abmad, wieso er so mit seinem Kind redet und wie seine Erziebung bis jetzt gewesen ist.

Spontan melden sich auch andere Stimmen zu Wort:

"Also ich sehe nur Dominanz. Da wird nicht nachgefragt, warum spielst du? Nur Vorwürfe, ein Gewitter an Ansagen. Es wird ibm ein schlechtes Gewissen gemacht, mehr nicht. Da ist null Interesse für den Sohn."

"Ich kenne das von meinem Vater, die Erniedrigungen, die Vorwürfe. Das hat mir keinen Spaß gemacht. Ich war einfach nicht religiös und hab mir das dann einfach nur angehört. Ich konnte ja nicht einfach meinen Vater schlagen, auch wenn ich ..."

"Und dann bist du auf die Straße gegangen und hast andere dafür geschlagen. Stimmt's?", sagt ein Mitgefangener. Stille.

Ein anderer redet weiter:

"Was soll er denn auch tun? Den Vater kritisieren? Das macht man doch nicht. Wenn ich öfter auf meinen Vater gebört bätte, säße ich heute nicht hier. Ganz bestimmt nicht."

"Aber da kommt nur Kritik. Ich sehe keine Liebe. Meine Eltern haben mir das auch nicht beigebracht. Ich habe irgendwann gemerkt, dass mir etwas feblt. Deshalb möchte ich das ändern. Ich möchte lernen, über meine Gefüble zu sprechen. Ich mache deshalb eine Gewalttherapie."

Ich bin überrascht, wie sich diese Männer den anderen Gefangenen und uns gegenüber allmählich öffnen, über Fehler nicht nur nachdenken, sondern auch sprechen, sie zugeben, sie reflektieren. Und das, obwobl wir uns erst eine halbe Stunde kennen."s

Vertrauen und Verständnis sind Grundvoraussetzungen, um Adressaten des Projektes in einer für sie schwierigen Phase zu erreichen und keine belehrende Auseinandersetzung um kulturelle und religiöse Einstellungen. Ein aufgeklärtes und mündiges Leben müssen die inhaftierten Männer selbst führen, die Impulse für die Voraussetzung dafür, ein einfühlsames und kritisches Hinterfragen, geben die Workshop-Leiter. Gleichzeitig entziehen sie so den Nährboden für die Entwicklung von radikalen und extremen Einstellungen, die als Anknüpfungspunkte für islamistische Ansprache in Frage kommen.

\section{Theoretisch-empirischer Hintergrund}

Islamismus ist, wie auch der Faschismus, für eine plurale und freiheitliche Gesellschaft eine Bedrohung und mit großen Herausforderungen verbunden. Seien es legalistische und politisch subversiv agierende Akteure wie die Muslimbruderschaft oder der Sala-

5 Ebd. Aus Mansour, Ahmad (2018): Klartext zur Integration. Gegen falsche Toleranz und Panikmache. Frankfurt am Main: S. Fischer, S. $12 \mathrm{ff}$. 
fismus als eine fundamentale Spielart des Islamismus, sie alle üben eine nicht zu unterschätzende Anziehungskraft vor allem auf Jugendliche und junge Erwachsene aus (vgl. Dantschke, Mansour, \& Müller, 2011). Allmähliche Radikalisierungsprozesse sind anfänglich nicht prinzipiell mit einer Sprache der Gewalt verbunden und werden daher häufig erst spät wahrgenommen. Aber sie bilden den demokratiefeindlichen Nährboden, in den der Samen für menschenverachtende Weltbilder für politische Ambitionen gesät werden (vgl. bpb, Gaisbauer, Jung, 2018, S. 146 f.). Setzen wir erst dort an, wo der Islamismus schon sich in gewalttätigen Handlungen gezeigt hat oder in diese übergeht, ist unterlassene Präventionsarbeit kaum mehr wiedergutzumachen.

Um Entscheidungsmuster junger Menschen für radikale religiöse Ideologien und ihre Verhaltenseinstellung nachvollziehen zu können, betrachten wir Radikalisierungsmechanismen hauptsächlich auf drei Ebenen, um zu verstehen, aus welchen wesentlichen sozialen Verhältnissen und biographischen Entwicklungen heraus Jugendliche überaus interessiert werden für islamistische Ideologien.

\section{Die psychologische Ebene}

Das sind psychischen Zustände eines Individuums, die sowohl veranlagt sein können als auch durch äußere soziale Einflüsse entstehen. Häufig sind das Jugendliche mit problematischen narzisstischen Persönlichkeitsstrukturen und Depressionen. Kritische Lebensereignisse, wie den Tod einer nahestehenden Person, Arbeitslosigkeit, $\mathrm{Zu}$ kunftsängste oder die eigene Inhaftierung katapultieren diese Heranwachsende häufig in eine emotionale Extremsituation, die sie kaum ertragen können (vgl. ebd., S. 208).

Die sozialen Beziehungen einer Person stellen weitere immens wichtige Faktoren dar. Fehlen familiäre Strukturen oder sind äußerst schwierig, fehlt die soziale Anbindung, kommen Mobbing in Schulen und Diskriminierungserfahrung hinzu, bestärkt dies die Unzufriedenheit und die Verzweiflung über die eigene Lage (vgl. Aslan et al., 2018, S. 23ff.). Besonders brisant wird es bei Jugendlichen, die in patriarchalen Strukturen aufwachsen und/ oder eine Vaterfigur vermissen. Fehlt im patriarchalisch geprägten Haushalt diese entscheidende, wegweisende Instanz, fehlt es speziell männlichen Jugendlichen an einer Vorbildfigur. Um diese Krise zu beenden, suchen sie nach einem Ersatz, nach Bindungen, die ihnen einen festen Halt geben. Dann sind diese für islamistische Werbung besonders anfällig, die ihnen eine emotionale Bindung und starke soziale Beziehungen unter einer heiligen Mission verspricht (vgl. Logvinov, 2017, S. 39, Marwan Abou Taam, 2014, S. 250 und Dantschke, 2014, 200 f.).

Genau an dieser Stelle zeigen radikale Bewegungen, wie Islamismus und Rechtsradikalismus gemeinsame Schnittstellen: ihre Befähigung alternative soziale Entwürfe und Gewaltaffinität zu generieren. Sie präsentere sich als die neue auserwählte Familie, die ihren Mitgliedern Weltsicht, Sprache und Lebensstil oktroyieren (vgl. Logvinov, 2017, S. 113). 
Die soziologische Ebene

Die soziologische Betrachtung liefert weitere Risikofaktoren, die eine Radikalisierung befeuern können, dazu gehört besonders das Phänomen der Wahrnehmung des Globalisierungsprozesses (vgl. Biene et al., 2016, 29 und Aslan et. Al, 2018, 35). Sie geht für junge Menschen mit einem Gefühl der totalen Entgrenzung einher, die tradierte Muster der Weltinterpretation, der Orientierung und der Identität zunehmend auflöst und viele mit dem Gefühl zurücklässt, die politische Lage werde stets instabiler, Die als komplex und undurchsichtig erscheinende, sich ständig wandelnde Welt weckt das Bedürfnis nach einfachen Antworten auf vielschichtige Fragen. So haben deshalb neben Religionen auch Verschwörungstheorien derzeit Hochkonjunktur (vgl. Aslan et al., 2018, S. 24, S. 255 ff.).

Polarisierte und dichotome Weltbilder - einfache schwarz-weiß Darstellungen - reduzieren Komplexität und begeistern Jugendliche. Radikale Muslime arbeiten mit klaren, simplifizierenden Plausibilitäten und identitätsstiftenden Haltungen. Auf diese Weise können Darstellungen und elitäre Wahrheitsansprüche etabliert werden, die innerhalb der Gruppe ein Machtgefühl erzeugen und mit einem verstärkten Sendungsbewusstsein einhergehen: wie beispielweise, Muslime seien die besseren von Allah auserkorenen Menschen (vgl. Dantschke et al., 2016, S. 86 ff.)!

Eine aktuelle Studie von Bernard Rougier (2020) belegt, wie in Frankreich allmählich ganze Stadtviertel von radikalen Muslimen unter ihre Kontrolle geraten. Die Feldstudien hierzu veranschaulichen, wie dogmatische, konservative gegen die Aufklärung gerichtete Normen in den muslimischen Gemeinden immer mehr an Bedeutung gewinnen. Gleichzeitig nimmt der Einfluss des Staates bzw. der Stellenwert demokratischer und paritätischer Überzeugungen ab. In der Studie wird deutlich, dass auch wenn der Anteil der militanten und radikalen Muslime eine Minderheit darstellt, sie aber deutlich das religiöse Leben in den Gemeinden dominieren (vgl. Rougier, 2020).

Für Jugendliche mit hybriden Identitäten sind entsprechende ideologische Offerten besonders verlockend. Ein junger Deutscher mit türkischem Migrationshintergrund beispielsweise, der in Deutschland als „der Türke“ und in der Türkei oder sogar in seiner Familie als „der Deutsche“ wahrgenommen wird - findet durch die Ideologie des Islamismus eine alternative Identität, eine sichtbare, starke und eindimensionale Identität, die für ihn oder sie sehr attraktiv wirken kann. Dadurch grenzt er sich von der Mehrheitsgesellschaft und seiner Familie ab (vgl. Mafaalani \& Toprak, 2013, S. 35).

\section{Die ideologische Ebene}

Die ideologische Ebene ist in unseren Augen der wichtigste und der am wenigsten beachtete Einflussfaktor. Um ein adäquates und umfassendes Verständnis über die Problematik der Entstehungskontexte für Radikalisierung zu erhalten, ist es unabdingbar in aller Klarheit auszusprechen, dass Radikalisierung und Islamismus mit einem bestimmten Islamverständnis verschränkt sind. Dieses Islamverständnis hat sein Funda- 
ment auch in den religiösen Quellen, die auch für das „Mainstream-Islamverständnis“ verbindlich sind (vgl. Aslan et al., 2018, S. 43).

Wichtige kritikwürdige Eckpunkte dieser Islamverständnisse sind folgende Aspekte:

\section{Der Buchstabenglaube}

Innerhalb der islamischen Communities fehlt es an einer weit verbreiteten Offenheit und Flexibilität gegenüber Auslegungen, die den Koran im lokal-historischen Kontext interpretieren. Legalistische und dogmatische Exegesen, wie sie unter Salafisten vorherrschen, sowie konservative Islamverständnisse unterstützen die Entmündigung der Gläubigen. Die Verantwortung für Entscheidungen wird von der Aussage heiliger Texte abhängig gemacht, eine kritische, hinterfragende Haltung verhindert (vgl. Mansour, 2019, 128 und bpb, Gaisbauer, Jung, 2018, S. 208).

\section{Angstpädagogik}

Angst ist ein zutiefst verstörendes und verunsicherndes Instrumentarium im Islam sowie in anderen Religionen, um Gläubige gefügig zu machen. Die Angstpädagogik ist ein besonderes Instrumentarium der Disziplinierung und so ent- und besteht der Glaube nicht aus Überzeugung, sondern durch die Angst vor der göttlichen Bestrafung (vgl. Nordbruch et al., 2014, S. 368).

Die auf Furcht basierenden Muster lassen sich in den patriarchalen Strukturen vieler Muslime wiederfinden: Es ist der Vater, der die Regeln bestimmt und bei Ungehorsam (körperlich) bestraft. Während im Diesseits die körperliche Strafe in der Regel als direkte Reaktion auf das Fehlverhalten folgt, verhängt Gott im Jenseits ungleich größere Strafen. Den Gläubigen begleitet im Diesseits ein ständiges Schuld- und Angstgefühl: du kannst zwar in diesem Leben sündigen,- du wirst aber im Jenseits für alles bezahlen. Die Hölle schwebt ständig wie ein Damoklesschwert über den Gläubigen - obne Hölle kein Islam (vgl. Schneiders, 2017, 452)!

Die installierten Mechanismen der Angstpädagogik verhindern eine selbstbestimmte und freie Entscheidung des Individuums, sich bewusst zum Islam zu bekennen. Das Recht auf die Entwicklung zum mündigen Denken wird dem Gläubigen vorenthalten. Die Angst vor Allah unterbindet eine mögliche lokal-historische, an der heutigen Gesellschaft orientierte Koranexegese und errichtet Anknüpfungspunkte für „religiöse Autoritäten“, um abweichende Meinungen wieder auf Linie zu bringen oder Gläubige gar davon abzuhalten, einen liberalen und individuellen Lebensstil einzuschlagen.

Diese Grundhaltung im Mainstream-Islam stellt für viele Muslime in Deutschland Integrationshürden auf: aus Angst vor „Sünden“, die die westliche Welt offenbart wie beispielweise die Gleichberechtigung von Mann und Frau, die sich unter anderem im Kleidungsstil äußert - wird der Prozess der Integration (z. B. das emotionale Ankommen), zusätzlich durch die Rhetorik der Angst erschwert bzw. unmöglich (vgl. Aslan, 2018, S. 63). 
Antisemitismus und Opferrolle

Der religiös-politisch begründete Hass auf Juden ist ein spezifischer Katalysator, der auf der ideologischen Ebene die Radikalisierung junger Muslime befördert. Antisemitismus - er ist zwar herkunftsübergreifend - wird aber gerade in salafistischen Ideologien offen zur Schau gestellt und ist zentraler Bestandteil der Welterklärung: Es seien gerade die von Gott verfluchten Juden, die als Feinde Allahs seine Religion des Islam und die Umma aus Rache vernichten wollten. Dafür hätten sie ein weltweites Netz an Intrigen gespannt und seien schuld an der Miesere in der islamischen Welt.

Das ist ein oft im Elternhaus oder durch sonstige soziale Kontakte tradiertes Feindbild „der Juden“, sie wird bewusst genutzt, um den Islam/die Muslime in eine Opferrolle zu manövrieren. Dabei wird er durch buchstabengetreuen Glauben der heiligen Schriften religiös gefüttert, ohne sie im historischen und lokalen Kontext zu verstehen. Bilder und Videos spielen in der jugendlichen Popkultur bewusst mit dem „Feindbild der Juden". Dabei sparen die Verfasser nicht an Verschwörungstheorien, nach denen "die“ Juden bereits die ganze Welt beherrschen oder sogar hinter Terroranschlägen stecken, die sie den Muslimen zuschieben würden (vgl. Marz, 2014).

\section{Methodik-Eine Anleitung zur Mündigkeit}

Im Hinblick auf die außerordentliche Bedeutung der Präventionsarbeit in diesem Kontext geht es nicht einfach darum, bei der Radikalisierung Jugendlicher, den nächsten Terroranschlag zu verhindern. Das ist natürlich wichtig, aber vor allem müssen wir die Wurzeln der Radikalisierung bekämpfen - durch durchdachte Präventionsarbeit.

\section{Das Ziel von Präventionsarbeit ist Mündigkeit}

Oberste Priorität der Präventionsarbeit bezweckt die pädagogische Hinführung zur Mündigkeit, denn wer selbstständig kritisch zu reflektieren vermag, wird in der Regel nicht radikal. Oft aber wachsen junge Menschen in gebieterischen und intoleranten Verhältnissen auf. Ihr Leben bestimmen patriarchale und althergebrachte kollektive Lebensstile, die Strukturen etablieren, die einer demokratischen Partizipation an der Gesellschaft diametral gegenüberstehen. Nicht Hinterfragung und Selbstbestimmung, sondern Gehorsam ist die einzige Option, die ihnen verbleibt.

Aus diesen Gründen gilt es, diese jungen Menschen zu eigenen Urteilsfindungen zu bringen. Sie sollen kritisches Denken lernen, um sie damit mündiger im Umgang mit Themen wie patriarchalischen Erziehungsstrukturen, Antisemitismus, Geschlechterrollen oder Verschwörungstheorien zu machen, die bei Radikalisierungsprozessen als Risikofaktoren wirken. Folgende notwendige Aspekte sind als Richtlinien für wirkungsvolle Präventionsarbeit erforderlich: 
1. Präventionsarbeit muss professionell und daber auch religiös neutral sein.

2. Die Arbeit muss durch gut ausgebildete Pädagogen, Sozialarbeiter oder Psychologen geleistet werden. Träger und Mitarbeiter müssen einer Sicherheitsprüfung durch den Staats- oder Verfassungsschutz unterzogen werden (Demokratie-Klausel).

3. Die Arbeit muss in erster Linie dazu dienen, die Teilnebmer zu mündigen Menschen zu machen.

4. Die Arbeit muss nachbaltig sein.

5. Folgende Themen müssen Bestandteil der Präventionsarbeit sein:

a. Kritisches Denken und Hinterfragen muss gefördert werden.

b. Religiöse Dogmen, wie Buchstabenglaube, Exklusivitätsanspruch und Angstpädagogik, dürfen nicht vermieden werden. Sobald sie für die Arbeit Relevanz aufweisen, müssen sie angesprochen und kritisch hinterfragt werden.

c. Opfer-und Feindbilder müssen thematisiert und binterfragt werden.

d. Nahostkonflikt, Verschwörungstheorien und (religiöse) Argumente des Antisemitismus müssen ebenso angesprochen werden.

e. Meinungsfreibeit. Es gebt darum, das Debattieren zu erlernen, andere Meinungen und Argumente auszubalten und in der Gruppe zu diskutieren.

f. Religionsfreibeit - positiv und negativ: Es gilt, die Freibeit von Religionen sowie die Gleichberechtigung der Religionen zu thematisieren.

g. Rollenbilder, patriarchalische Strukturen, Tabuisierung von Sexualität und sexuelle Selbstbestimmung.

6. In den Einrichtungen, in denen Präventionsarbeit stattfindet, wie Schulen, Jugendzentren oder Justizvollzugsanstalten, müssen auch die übrigen Mitarbeiter Zugang zu dieser Arbeit haben. Sie müssen miteinbezogen werden oder als Beobachter teilnebmen dürfen.

7. Präventionsarbeit darf nicht „,bemuttern“, sondern sie soll intellektuell herausfordern. Sie darf nicht belehrend sein, sondern muss auf Augenhöhe stattfinden. Beispielsweise durch Imame, die von vorneherein als Respektspersonen gelten und in der patriarchalischen Struktur ganz oben steben, sind viele junge Menschen ein solches Diskussionsverbältnis nicht gewohnt. Ihnen zu vermitteln, Gott wolle dies, jenes aber nicht, mag für den Moment dazu geeignet sein, sie zum Nachdenken und von Gewalt abzubringen. Auf Dauer werden durch diese Bevormundung aber keine mündigen Menschen entsteben, die selbst denken und handeln. Dies birgt jedoch die Gefabr, dass über Autoritätspersonen, die ibre Position missbrauchen, auch in Zukunft ein Einfallstor für die Vermittlung von Inbalten geöffnet bleibt, die als Risikofaktoren für eine Radikalisierung wirken könnten (z. B. Angstpädagogik, Buchstabenglaube, Tabuisierung von Sexualität, Opfer-und Feindbilder usw.).

Die methodische Umsetzung des Angebots von MIND prevention stützt sich vor allem auf das Rollenspiel. Sie ermöglicht spielerisch eine emotionale und affektive Auseinandersetzung sowie einen leichten und unterhaltsamen Zugang zu den Themen. Konfliktsituationen werden nicht nur anschaulich in Szene gesetzt, sondern interaktiv 
mit den Jugendlichen weiterentwickelt. Wichtige konzeptionelle Bedingung ist daher, dass alle Workshopleiter einen pädagogischen Hintergrund haben und den gleichen religiösen wie kulturellen Hintergrund aufweisen wie die Teilnehmer.

Das Rollenspiel als inszenatorische Vorgehensweise eignet sich sehr gut dazu, um die Thematik und den Handlungsspielraum optimal zu verinnerlichen, denn die Teilnehmer werden in die Diskussion aktiv involviert. Sie werden ermutigt, Fragen zu stellen, zum Teil beantworten sie die Fragen selbst. Sie bestimmen die Verlaufsdynamik des Workshops selbst, in dem sie selbst Konventionen und das eigene Gedankengut aktiv gegenseitig hinterfragen und gemeinsam neue Perspektiven entwickeln (vgl. Freudenreich et al., 1980; Freudenreich \& Sperth, 1983; Ments, 1985).

Die Rollenspiele sind dabei niemals in sich geschlossene moralische Geschichten, sondern sie rekurrieren auf alltägliche Situationen dieser Menschen. Die WorkshopLeiter nehmen keine belehrende Funktion ein, was die Lektion sein soll, diese handeln die Teilnehmer begleitet von den Workshopleitern im Dialog aus.

Es bleibt jedoch nicht bei der alleinigen Diskussion der Inhalte. Im nächsten Schritt sollen die Teilnehmer eingebunden, neue Fragen oder ihre Anregungen, andere Perspektiven wunschgemäß in Szene gesetzt werden. Hierbei setzen die Teilnehmer, die im Workshop erarbeiteten und vorgeschlagenen Lösungsansätze zum Teil selbst durch. Es ist ja das dezidierte Ziel, diese Menschen zum selbstständigen Denken und Handeln zu ermutigen.

Schließlich wird eine weitere positive Auswirkung - der Multiplikatoreneffekt - angestrebt. Die Vermittlung von Wissen von den geschulten Workshop-Leitern als Peers soll auf die Teilnehmer übergehen, so dass diese wiederum in ihren betreffenden Peergruppen als Wissensvermittler eine Rolle als Multiplikator einnehmen können.

Workshops, die theaterpädagogische und systemische Methoden beinhalten, haben sich in analogen Kontexten bewährt. Teilnehmer solcher Workshops können sich erproben, verschiedene Blickwinkel einnehmen und verschiedene Darstellerpositionen einnehmen. Der Wechsel zwischen Akteurs- und Beobachterperspektive erweitert den gedanklichen Horizont, verinnerlichte Strukturen werden für einen Moment verlassen und andere Betrachtungswinkel eingenommen. Dies stärkt zuletzt die Reflexionsfähigkeit der an solchen Workshops Teilnehmenden.

Im Rollenspiel versetzen sich die Beteiligten emotional in andere Betrachtungslagen, dies ermöglicht kritische Narrative neu zu besetzen bzw. Gegennarrative zu erarbeiten. So können für alltägliche Konfliktsituationen und für Themen, aus denen sich Radikalisierungsprozesse entzünden könnten, Lösungswege verdeutlicht werden. Mit einer gesteigerten Fähigkeit zur Problemlösung geht auch eine wirksame Einstellungsänderung einher. Aus diesem Grund werden derartige Rollenspiele nach der sogenannten Fischteichmethode durchgeführt, wobei ein Kreis aus Beobachtern die spielenden Akteure umringt und sich jederzeit in die Handlung involvieren kann (vgl. Fritz et al., 2019, S. 197).

Die Workshops bezwecken nicht, den Teilnehmern eine vorgefertigte Wahrheit zu präsentieren, vielmehr sollen Denkprozesse angestoßen werden. In diesen geht es nicht um theologische Auslegungen oder Argumentation, wie Gottes Wille zu deuten ist, 
sondern die Jugendlichen selbst auf Ideen und Alternativen kommen zu lassen. „Nur wer selbst denkt, ist mündig - Freiheit beginnt im Kopf“.

Eine weitere wichtige Richtlinie der Workshop Umsetzung lautet: „Wir greifen niemanden in seinem Glauben an“. Vielmehr soll ein Raum für Gedankenspiele entstehen und Meinungen anschließend mit der Methode des begleiteten Entdeckens in Frage gestellt werden, ohne dabei die Meinung der Jugendlichen zu verurteilen.

Angebote dieser Art befinden sich an der Schnittstelle von primärer (richtet sich an alle) und sekundärer Präventionsarbeit (richtet sich an Personen mit Risikofaktoren). Das Vorgehen unterscheidet sich in Abhängigkeit davon, an welchem Punkt der Adressat sich auf der Zeitachse eines Radikalisierungsprozesses befindet. Im Wesentlichen muss unterschieden werden, ob es sich um Präventions- oder um Deradikalisierungsarbeit handelt. Im ersten Fall ist das Ziel die Vorbeugung einer potenziellen Radikalität, weshalb die Zielgruppe hier auch viel weiter gefasst werden muss als im zweiten Fall, in dem das sprichwörtliche Kind schon in den ideologischen Brunnen gefallen ist.

\section{Diskussion - Aufklärung E Emanzipation statt muslimischer Identitätspolitik}

Die Diskussion um eine effektive Prävention islamischer Radikalisierung ist längst ein Politikum und erschwert eine angemessene Problemdiagnose, wie auch die Wege zu nachhaltigen Lösungen. Es ist nicht die theologische Aufgabe von Präventionsträgern darüber aufzuklären, ob nun das Kopftuch im Islam eine Pflicht sei oder ob Gebetsräume in Schulen ein besseres Klima des Miteinanders schaffen können. Auf diesen Zug sind längst andere aufgesprungen, die islamischen Radikalismus aus einer Opferrolle der Betroffenen erklären und in diesem Zusammenhang Islamfeindlichkeit und unerfüllte Konzessionen für religiöse Befindlichkeiten als Motive für Radikalisierung feststellen. Dabei liegen die Ursachen für religiös bedingte Menschenfeindlichkeit ganz wo anders.

Innerhalb der wissenschaftlichen Debatte um die Gründe zur Hinwendung Jugendlicher zum islamistischen Radikalismus ist der französische Diskurs interessant und symptomatisch für den Grundtenor, wie das Problem islamistischer Terrorismus behandelt wird. Zwei Erklärungsansätze stehen dabei gegenüber. Die eine Interpretation, vertreten vom französischen Politikwissenschaftler und Islamismus-Experte Oliver Roy, geht von einer Islamisierung der Radikalisierung aus (vgl. Roy, 20017, S. 16, 20). Terroristische Anschläge hätten nicht ihren Ursprung im Islam, sondern wären vielmehr Ausdruck eines Jugendaufstands. Radikalismus würde junge Menschen faszinieren: „Sie sind radikal, weil sie radikal sein wollen, weil sie die schiere Radikalität verlockend finden. Egal welche Daten man auch heranzieht, die mangelhafte religiöse Bildung der Dschihadisten ist immer offensichtlich" (Roy, 2017, S. 70).

Eine konträre Meinung vertritt der Islamforscher Gilles Kepel, der Roy eine Verharmlosung der islamischen Dimension von Radikalismus und Terrorismus vorwirft. Der islamistische Radikalismus sei - in Frankreich - eine Reaktion auf Atheismus und 
den Laizismus, geschürt würde dieser zumal wegen der Kolonialgeschichte in Nordafrika (vgl. Kepel, 2017, Pos. 334, 692, Kindle-Edition). Er verweist auch auf die islamimmanente Ursache von Gewalt und Intoleranz indem er auf muslimische Parallelgesellschaften in den Banlieues und den zunehmenden Einfluss von Salafisten verweist, die immer mehr religiöse Vorgaben im öffentlichen Leben durchsetzen. ${ }^{6}$

Im Vergleich beider Interpretationsversuche für die Ursachen und für die Attraktivität des islamistischen Radikalismus lässt Oliver Roys Auslegung offen, warum gerade der Islam auf Radikale so anziehend wirkt. Sie vernachlässigt auch den Einfluss religiös initiierter Haltung im Hinblick auf die Einteilung der Welt in Gute und Böse, welche legitimen Gewaltmittel zur Durchsetzung religiöser Standpunkte deklariert werden und welche negativen Impulse für emanzipatorische Prozesse ausgehen.Für viele muslimische Jugendliche bzw. junge Menschen ist es aufgrund des in islamischen Communities und in den Familien tradierten Islamverständnisses nicht möglich, einen affirmativen und positiven Bezug zu den demokratischen Werten westlicher Gesellschaften aufzubauen. In Folge entstehen Parallelgesellschaften, in denen keinerlei Motivation für eine nachhaltige Integration in die plurale Werteordnung einer nicht-muslimischen Mehrheitsgesellschaft vorhanden ist. Auch wenn der agitatorische Einfluss islamistischer Gruppierungen in jüngster Zeit geschwunden zu sein scheint, finden sich hier immer noch die meisten potenziell gewaltaffinen Anhänger für die Ideologie des Islamismus (vgl. Tibi, 2019, S. 28).

Eine effektive und erfolgreiche Präventionsarbeit muss vor diesem Hintergrund eine klare und deutliche Sprache sprechen und gerade die aufgezählten Themen offen ansprechen, um Radikalisierungspotenziale zu vermeiden bzw. zu mindern. Das muss ohne Scheu vor religiösen Wohlfühlzonen geschehen, aber pädagogisch mit Empathie und Respekt. Der Weg zu einer mündigen und aufgeklärten Haltung ist besonders für Jugendliche ein schwerer und steiniger Weg. Oft begleitet von Schuldgefühlen unter dem Auge konservativer Familienstrukturen oder der "Glaubens-Community“. Hier gilt es Irritationen zu erzeugen, diese aufzufangen und in kritische Reflektionsprozesse überzuleiten. Keine leichte Aufgabe, aber eine machbare und erforderliche. Radikale Weltbilder und Unmündigkeit als Ohnmacht zu demaskieren, Mündigkeit und kritisches Denken als Stärke zu vermitteln, das sind oberste Prioritäten einer Präventionsarbeit.

\section{Literaturverzeichnis}

Aslan, E., Erşan Akkıliç, E. \& Hämmerle, M. (2018). Islamistische Radikalisierung. Biografische Verläufe im Kontext der religiösen Sozialisation und des radikalen Milieu (Wiener Beiträge zur Islamforschung). Wiesbaden: Springer VS.

6 https://www.tagesanzeiger.ch/ausland/europa/der-jihadismus-will-europa-in-einen-buergerk rieg-stuerzen/story/16112643, aufgerufen am 6.1.2019. 
Biene, Janusz, Christopher Daase, Julian Junk und Harald Müller (Hrsg.) (2016), Salafismus und Dschihadismus in Deutschland. Frankfurt am Main: Campus.

Broich, J. (1994). Rollenspiele mit Erwachsenen (5. Aufl.). Köln: Maternus.

Bundeszentrale für politische Bildung/bpb, Gaisbauer, S. \& Jung, M. (2018). Dossier "Islamismus". Bonn: Bundeszentrale für politische Bildung.

Dantschke, C. (2014). Radikalisierung von Jugendlichen durch salafistische Strömungen in Deutschland. In R. Ceylan \& B. Jokisch (Hrsg.), Salafismus in Deutschland. Entstehung, Radikalisierung und Prävention (ROI - Reihe für Osnabrücker Islamstudien, Bd. 17, 1st, New ed., S. 193-214). Frankfurt a.M: Peter Lang GmbH Internationaler Verlag der Wissenschaften.

Dantschke, C., Mansour, A. \& Müller, J. (2011). "Ich lebe nur für Allah". Argumente und Anziehungskraft des Salafismus; eine Handreichung für Pädagogik, Jugend- und Sozialarbeit, Familien und Politik; Modellprojekt "Demokratie stärken - Auseinandersetzung mit Islamismus und Ultranationalismus" der ZDK Gesellschaft Demokratische Kultur gGmbH (Schriftenreihe Zentrum Demokratische Kultur).

Dantschke, C., Taam, M. A., Kreutz, M. \& Sarhan, A. (2016). Anwerbungspraxis und Organisationsstruktur. In Biene, Janusz, Christopher Daase, Julian Junk und Harald Müller (Hrsg.), Salafismus und Dschihadismus in Deutschland (S. 79-116). Campus. Janusz Biene, Christopher Daase, Julian Junk und Harald Müller

Freudenreich, D. \& Sperth, F. (1983). Stundenblätter Rollenspiele im Literaturunterricht. Sekundarstufe I (Klett/Schulpraxis, 1. Aufl.). Stuttgart: Klett.

Freudenreich, D., Gräßer, H. \& Köberling, J. (1980). Rollenspiel. Rollenspiellernen für Kinder und Erzieher in Kindergärten, Vorklassen und ersten Schuljahren; 126 Spielvorlagen (Schroedel elementar Praxis Handbuch, 4., überarb. u. erw. Aufl.). Hannover: Schroedel.

Fritz, U., Lauermann, K., Pächter, M., Stock, M. \& Weirer, W. (2019). Kompetenzorientierter Unterricht: Theoretische Grundlagen - erprobte Praxisbeispiele: UTB $\mathrm{GmbH}$.

Kepel, Gilles (2017). Der Bruch. Frankreichs gespaltene Gesellschaft. München: Verlag Antje Kunstmann. Kindle-Version.

Logvinov, M. (2017). Salafismus, Radikalisierung und terroristische Gewalt: Erklärungsansätze - Befunde - Kritik: Springer Fachmedien Wiesbaden.

Mafaalani, A. e. \& Toprak, A. (2013). Muslimische Kinder und Jugendliche in Deutschland. Lebenswelten - Denkmuster - Herausforderungen (Eine Veröffentlichung der Konrad-Adenauer-Stiftung e.V, 2., unveränd. Aufl.). Sankt Augustin: Konrad-Adenauer-Stiftung.

Mansour, A. (2019). Eine nationale Strategie gegen die Radikalisierung. In C. Linnemann \& W. Bausback (Hrsg.), Der politische Islam gehört nicht zu Deutschland. Wie wir unsere freie Gesellschaft verteidigen (S. 122-141). Freiburg: Verlag Herder.

RPsych 6. Jg. 1/2020 
Mansour, Ahmad (2018): Klartext zur Integration. Gegen falsche Toleranz und Panikmache. Frankfurt am Main: S. Fischer.

Marz, U. (2014). Kritik des islamischen Antisemitismus: Zur gesellschaftlichen Genese und Semantik des Antisemitismus in der Islamischen Republik Iran: Lit.

Ments, M. Van (1985). Rollenspiel: effektiv. E. Leitf. für Lehrer Erzieher Ausbilder u. Gruppenleiter (EGS-Texte). München: Ehrenwirth.

Müller, Jochen; Götz Nordbruch, Aladin El-Mafaalani, Alma Fathi, Ahmad Mansour, Julian Waleciak, Dantschke, C., Mansour, A. \& Müller, J. (2011). "Ich lebe nur für Allab". Argumente und Anziebungskraft des Salafismus; eine Handreichung für Pädagogik, Jugend- und Sozialarbeit, Familien und Politik; Modellprojekt "Demokratie stärken - Auseinandersetzung mit Islamismus und Ultranationalismus" der ZDK Gesellschaft Demokratische Kultur gGmbH (Schriftenreihe Zentrum Demokratische Kultur).

Deradikalisierung (2016). Prävention und Deradikalisierung. In Janusz Biene, Christopher Daase, Julian Junk und Harald Müller (Hrsg.), Salafismus und Dschihadismus in Deutschland (S. 233-270). Frankfurt am Main: Campus.

Nordbruch, G., Müller, J. \& Ünlü, D. (2014). Salafismus als Ausweg? Zur Attraktivität des Salafismus unter Jugendlichen. In T. G. Schneiders (Hrsg.), Salafismus in Deutschland. Ursprünge und Gefahren einer islamisch-fundamentalistischen Bewegung (Globaler lokaler Islam, S. 363-372). Bielefeld, Germany: transcript.

Roy, Olivier (2017): "Ihr liebt das Leben, wir lieben den Tod". Der Dschihad und die Wurzeln des Terrors. Erste Auflage. München: Siedler.

Rougier, B. (2020). Les territoires conquis de l'islamisme. Paris: Presses Universitaires de France (PUF).

Schneiders, T. G. (Hrsg.). (2014). Salafismus in Deutschland. Ursprünge und Gefahren einer islamisch-fundamentalistischen Bewegung (Globaler lokaler Islam). Bielefeld, Germany: transcript. https://doi.org/10.14361/transcript.9783839427118

Taam, M. A. (2014). Radikalisierungsmechanismen am Beispiel des Salafismus in Deutschland. In R. Ceylan \& B. Jokisch (Hrsg.), Salafismus in Deutschland. Entstehung, Radikalisierung und Prävention (ROI - Reihe für Osnabrücker Islamstudien, Bd. 17, 1st, New ed., S. 239-254). Frankfurt a.M: Peter Lang GmbH Internationaler Verlag der Wissenschaften.

Tibi, B. (2019). Wie die Integration islamischer Zuwanderer nach Europa behindert wird. In C. Linnemann \& W. Bausback (Hrsg.), Der politische Islam gehört nicht zu Deutschland. Wie wir unsere freie Gesellschaft verteidigen (S. 24-41). Freiburg: Verlag Herder. 


\title{
Kontakt:
}

\author{
Ahmad Mansour, \\ Geschäftsführung MIND prevention, \\ @: ahmad.mansour@mind-prevention.de \\ Dr. phil. Gürcan Kökgiran, \\ ReThink Projektkoordination, \\ @: guercan.koekgiran@mind-prevention.de
}

